\title{
HLA-B*5701 frequency in Chilean HIV-infected patients and in general population
}

\author{
Author \\ Helena Poggi ${ }^{1}$ \\ Alejandra Vera ${ }^{1}$ \\ Marcela Lagos ${ }^{1}$ \\ Sandra Solari ${ }^{2}$ \\ Luis Rodríguez $\mathrm{P}^{3}$ \\ Carlos M Pérez \\ ${ }^{1}$ Molecular Biology \\ Laboratory, Department of \\ Clinical Laboratories, \\ School of Medicine, \\ Pontificia Universidad \\ Católica de Chile, Santiago, \\ Chile. \\ ${ }^{2}$ Toxicology Laboratory, \\ Department of Clinical \\ Laboratories,School of \\ Medicine, Pontificia \\ Universidad Católica de \\ Chile, Santiago, Chile. \\ ${ }^{3}$ Department of Clinical \\ Laboratories,School of \\ Medicine, Pontificia \\ Universidad Católica de \\ Chile, Santiago, Chile. \\ ${ }^{4}$ Department of Internal \\ Medicine and Program of \\ Infectious Diseases,School \\ of Medicine, Pontificia \\ Universidad Católica de \\ Chile, Santiago, Chile.
}

Submitted on: 03/04/2010 Approved on: 06/01/2010

\section{Correspondence to: \\ Helena Poggi \\ Laboratorio Biología \\ Molecular \\ Vicuña Mackenna 4686 \\ Santiago de Chile \\ Chile \\ E-mail: hpoggi@ \\ med.puc.cl}

We declare no conflict of interest.

\begin{abstract}
It has been demonstrated that HLA-B ${ }^{\star} 5701$ screening reduces the risk for hypersensitivity reaction to abacavir in $\mathrm{HIV}$-infected patients. Since $\mathrm{B}^{\star} 5701$ prevalence varies among different populations, it is important to determine the carrier frequency prior to its use for the screening of HIV-infected patients. The aim of this study was to determine HLA-B $5^{2} 701$ carrier frequency in Chilean general population and HIV-infected patients referred for $\mathrm{B}^{\star} 5701$ typing. For that purpose 300 blood bank donors and 492 abacavir-naïve HIV-infected patients from Chile were screened for $B^{\star} 5701$ by a sequence specific primer PCR. We detected 14/300 (4.7\%) $\mathrm{B}^{\star} 57$-positive individuals in the Chilean general population, $11(3.7 \%)$ were $\mathrm{B}^{\star} 5701$ positive, and $3(1 \%)$ had another subtype. All were heterozygous, thus a $B^{\star} 5701$ allele frequency of $2 \%$ was determined. Eleven of $492(2.2 \%) \mathrm{HIV}$-patients carried a B ${ }^{\star} 701$ allele. The difference between these frequencies is probably due to slow progression of HIV infection in HLA-B ${ }^{\star} 5701$ carriers, thus less patients would require antiretroviral therapy and $B^{\star} 5701$ typing. Considering the usefulness of $B^{\star} 5701$ screening, its prevalence in the Chilean general population, and the availability of a validated method, we conclude that HLA-B ${ }^{\star} 701$ typing in Chilean HIV-infected patients about to initiate abacavir treatment is strongly recommended.
\end{abstract}

Keywords: HLA-B ${ }^{\star} 5701$, HIV, abacavir, Chile, pharmacogenetics.

[Braz J Infect Dis 2010;14(5):510-512]@Elsevier Editora Ltda.

Abacavir is a nucleoside reverse-transcriptase inhibitor, with few drug interactions and a favorable safety profile, therefore commonly used in combination with other antiretroviral agents as part of highly active antiretroviral treatment regimens. Approximately 5\%-8\% of human immunodeficiency virus (HIV) infected patients on abacavir treatment develop a hypersensitivity reaction (HSR) which usually occurs within the first 6 weeks of treatment. ${ }^{1}$ HSR is characterized by a multisystemic involvement that becomes more severe with continuous dosing, requiring discontinuation of the drug. Subsequent rechallenge with abacavir must be avoided, since there is a high risk of developing a life threatening reaction. ${ }^{2}$ HSR incidence varies among different populations, being more common in Caucasian patients than in those of African or Asian origin. ${ }^{3}$ Abacavir HSR occurs in approximately $4 \%$ of Chilean HIV-infected patients (Chilean AIDS Cohort, Carlos Beltran personal communication).

Since 2002, many studies have reported a strong association between abacavir HSR and the class I human leukocyte antigen (HLA) allele $B^{\star 5701 ., 5}$
More so, the utility of HLA-B ${ }^{\star} 5701$ screening as a pharmacogenetic test to predict the risk of developing HSR has been demonstrated in abacavirnaïve HIV-infected patients. ${ }^{6,7}$ The incidence of HSR may vary substantially among different populations due to the heterogeneity in HLA-B ${ }^{\star} 5701$ prevalence across distinct ethnicities. ${ }^{3,8}$ Ideally, before implementing HLA-B ${ }^{\star} 5701$ genetic screening in a given population, its prevalence should be ascertained, particularly if it is unknown or poorly described, as in the Chilean population. Serological methods for HLA-B57 detection lack specificity and commercial molecular HLA-B typing methods used for identifying all known HLA-B alleles are expensive and laborious. Therefore the implementation of an alternate cost-effective method for HLA-B ${ }^{\star} 5701$ screening would be an appropriate approach to reduce the risk of developing HSR in the HIV positive patient population that is going to receive abacavir treatment. The aim of this study was to determine HLA-B ${ }^{\star} 5701$ carrier frequency in Chilean general population and HIV-infected patients after implementing a typing method for HLA-B ${ }^{\star 5701}$ screening. 
In order to validate the method for $\mathrm{B}^{\star} 5701$ typing, 28 External Quality Control DNA samples from the College of American Pathologists (CAP) HLA-typing survey ( $2 \mathrm{~B}^{\star 5701}$ positive, $26 \mathrm{~B}^{\star} 57$ negative), and $71 \mathrm{~B}^{\star} 57$ positive Chilean subjects (previously typed by a low resolution commercial method) were ana-

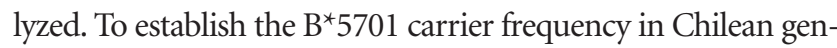
eral population, 300 unrelated anonymised blood bank donors (150 men and 150 women) were typed. After implementation and validation of HLA-B ${ }^{\star} 5701$ molecular typing for routine clinical testing, $492 \mathrm{HIV}$-positive abacavir-naïve patients were referred to our laboratory for HLA-B ${ }^{\star} 5701$ screening prior initiating abacavir treatment. DNA from all samples was extracted by a standard "salting out" method.

A touch down PCR was implemented using the sequence specific primers (SSP) and conditions described by Martin et al. ${ }^{9}$ with minor modifications. The forward primer was synthesized without the M13 sequence tag, and the PCR cycling conditions were slightly different as the first four cycles at $70^{\circ} \mathrm{C}$ annealing temperature were omitted. Also, a different target (the alpha-1-antitrypsin gen, AAT) was used as internal control of amplification, using the primers AAT3 (5'-CCC ACC TTC CCC TCT CTC CAG GCA AAT GGG-3') and AAT4 (5'-GGG CCT CAG TCC CAA CAT GGC TAA GAG GTG-3') at a final concentration of $2.5 \mu \mathrm{M}$. PCR products were subjected to electrophoresis on a $2 \%$ agarose gel stained with ethidium bromide and visualized under UV light. This multiplexed SSP-PCR allows the identification of alleles carrying the generic $B^{\star} 57$ and those with the subtype $B^{\star} 5701$ by their amplicon sizes: the HLA-B ${ }^{\star} 57$ group-specific primer pair amplifies a 175 bp fragment, HLA-B ${ }^{\star} 5701$-subtype specific primers a fragment of $94 \mathrm{bp}$, and the internal control primers a $360 \mathrm{bp}$ fragment. Given that this method only assesses the presence or absence of $\mathrm{B}^{\star} 57$ and $\mathrm{B}^{\star} 5701$ alleles, zygosity status could not be determined by this mean. Therefore a low resolution commercial typing method (Peel-Freez ${ }^{\circledR S S P}-$ UniTray ${ }^{\circledR}$ InvitrogenTM) was used to determine homozygosity or heterozygosity in the $B^{\star} 5701$ positive samples. Allele and carrier frequencies for HLA-B ${ }^{\star} 5701$ were determined by direct gene counting and the data from both populations (i.e. general population and HIV patients) were analyzed by using the Chi-square test $\left(\right.$ MINITAB $\left.^{\circledR} 14\right) .{ }^{10}$

The results obtained with the external quality control samples were $100 \%$ concordant with those reported by the CAP, and all $71 \mathrm{~B}^{\star 57}$ samples previously typed were correctly identified by the group-specific primers. Thus, this SSPPCR method was validated for routine analysis. See Figure 1 for typical results. The $\mathrm{B}^{\star} 5701$ allele was present in 64 of 71 (90\%) $\mathrm{B}^{\star} 57$ positive subjects, the 7 remaining (10\%) carried a different subtype. In the general population, from a total of 300 subjects analyzed, 14 individuals $(4.7 \%)$ carried a $\mathrm{B}^{\star} 57$ allele, from which 11 (3.7\%) were $B^{\star} 5701$, and $3(1.0 \%)$ had another subtype. The remaining 286 were negative for the
Figure 1: Typical SSP-PCR results. M: $100 \mathrm{bp}$ DNA size marker, S1: negative control for B*57 and B*5701 alleles, S2, S4, S6, and S7: samples positive for the B*5701 allele, S3 and S5: sample positive for $B * 57$ group-specific amplification, carrying a different subtype.

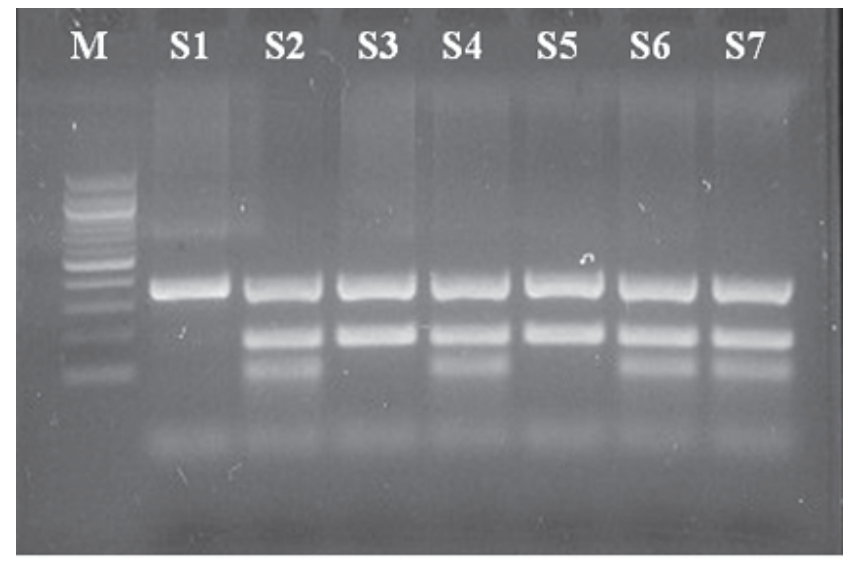

presence of this allele. All positive samples were heterozygotes, thus an allele frequency of $1.8 \%$ for the $\mathrm{B}^{\star} 5701$ allele, and of $0.5 \%$ for the $B^{\star} 57$ alleles with other subtypes was determined. Eleven (2.2\%) out of $492 \mathrm{HIV}$ patients were positive for the generic allele $B^{\star} 57$, all of them were of the subtype $\mathrm{B}^{\star 5701}$ and heterozygotes for this allele as determined by low resolution typing. None of the $\mathrm{B}^{\star} 5701$ positive HIV patients was subsequently treated with abacavir. The results are summarized in Table 1 . The observed $B^{\star} 5701$ carrier and allele frequencies were higher in the general population sample than in HIV-infected patients. The statistical analysis performed using the Chi-square test to determine if this difference between both populations was noteworthy, revealed that it was not significant $(\mathrm{p}<0.05)$.

A prospective screening genetic test should fulfill several requirements before its introduction into routine clinical practice, such as: improve the clinical outcome, have high predictive values, be cost-effective, and easy to implement technically. ${ }^{3}$ The clinical utility of $B^{\star} 5701$ screening is well demonstrated, so technical aspects and feasibility of an analysis method were important to be evaluated. The PCR-based technique implemented here for HLA-B ${ }^{\star} 5701$ screening proved to be $100 \%$ sensitive in detecting $B^{\star} 5701$ and $\mathrm{B}^{\star} 57$ positive alleles. It was also a method easy to implement and to validate, as well as technically simple to perform in a clinical routine setting. More so, an "in house" PCR, properly validated as this one, allows cost reduction when compared to commercial kits, thus allowing its use also in developing countries where less financial and technical resources are available.

The observed $B^{\star} 5701$ carrier frequency $(3.7 \%)$ in this general population sample from Chile is in agreement with data $(4.0 \%)$ from 70 Chilean individuals published at the "allele frequencies in worldwide populations" database. ${ }^{11}$ 
Table 1. HLA-B*57, B*5701 and other subtypes carrier and allele frequencies in Chilean population

\begin{tabular}{|c|c|c|c|c|c|c|}
\hline & \multicolumn{3}{|c|}{$\begin{array}{l}\text { General Chilean population } \\
\qquad \mathbf{n}=300\end{array}$} & \multicolumn{3}{|c|}{$\begin{array}{l}\text { Chilean HIV patients } \\
\qquad \mathbf{n}=492\end{array}$} \\
\hline & $\mathbf{n}$ & $\begin{array}{c}\text { Carrier } \\
\text { frequency }\end{array}$ & $\begin{array}{c}\text { Allele } \\
\text { frequency }\end{array}$ & $\mathbf{n}$ & $\begin{array}{c}\text { Carrier } \\
\text { frequency }\end{array}$ & $\begin{array}{c}\text { Allele } \\
\text { frequency }\end{array}$ \\
\hline B*57 (generic) & 14 & $4.7 \%$ & $2.3 \%$ & 11 & $2.2 \%$ & $1.1 \%$ \\
\hline B*5701 (allele specific) & 11 & $3.7 \%$ & $1.8 \%$ & 11 & $2.2 \%$ & $1.1 \%$ \\
\hline B*57_- (other subtype) & 3 & $1.0 \%$ & $0.5 \%$ & 0 & - & - \\
\hline
\end{tabular}

However, in this database there is no information about the status of those Chilean individuals (i.e. patients, controls or general population). Also, the $\mathrm{B}^{\star} 57$ carrier frequency published therein $(1.0 \%)$ is much different than the one observed by us (4.7\%), and unlikely to be correct, since the generic type of an allele cannot have a lower frequency than one of its subtypes. The fact that the $\mathrm{B}^{\star} 5701$ frequencies we observed in the general population and in the patient population samples are lower than the ones reported for Caucasians and higher than those in populations of Asian origin, is most likely due to our mixed genetic background of Caucasian and Native American of mongoloid ancestry. ${ }^{12,13}$

To our knowledge, there are no $B^{\star} 5701$ carrier or allele frequencies described for HIV-positive patients from other Latin American countries, except for Hispanics in the United States. ${ }^{3}$ The carrier frequency found in our HIV-infected patients (2.2\%) and in Chilean general population $(3.7 \%)$ were very similar to the ones reported for US-Hispanics. ${ }^{8}$ The difference between HIV-infected patients and general population, although not statistically significant, could probably be related to the finding that HLA-B ${ }^{\star} 5701$ carriage has been associated with slow progression of HIV infection, therefore less patients carrying a HLA-B ${ }^{\star} 5701$ allele would require antiretroviral therapy and thus $B^{\star} 5701$ typing. ${ }^{14,15}$ Considering the vast evidence on the benefits of $B \star 5701$ screening, its prevalence in the Chilean general population, and the availability of a proper screening method, $\mathrm{B}^{\star} 5701$ typing in Chilean HIV-infected patients about to initiate abacavir treatment is strongly recommended.

\section{REFERENCES}

1. Lucas A, Nolan D, Mallal S. HLA-B`5701 screening for susceptibility to abacavir hypersensitivity. J Antimicrob Chemother 2007; 59:591-3.

2. Hughes AR, Spreen WR, Mosteller M et al. Pharmacogenetics of hypersensibility to abacavir: from PGx hypothesis to confirmation to clinical utility. Pharmacogenomics J 2008; 8:365-74.

3. Phillips EJ. Genetic screening to prevent abacavir hypersensibility reaction: are we there yet? Clin Infect Dis 2006; 43:103-5.

4. Mallal S, Nolan C, Witt C et al. Association between presence of HLA-B ${ }^{\star} 5701$, HLA-DR7, and HLA-DQ3 and hypersensitivity to HIV-1 reverse-transcriptase inhibitor abacavir. Lancet 2002; 359:727-32.

5. Hetherington S, Hughes A, Mosteller M et al. Genetic variations in HLA-B region and hypersensitivity reactions to abacavir. Lancet 2002; 359:1121-1122.

6. Mallal S, Phillips E, Carosi G, et al. HLA-B ${ }^{\star} 5701$ screening for hypersensibility to abacavir. N Engl J Med 2008; 358:568-579.

7. Saag M, Balu R, Phillips E et al. High sensitivity of human leukocyte antigen- $B^{\star} 5701$ as a marker for immunologically confirmed abacavir hypersensitivity in white and black patients. Clin Infect Dis 2008; 46:1111-1118.

8. Hughes A, Mosteller M, Bansal A et al. Association of genetic variations in HLA-B region with hypersensitivity to abacavir in some, but not all, populations. Pharmacogenomics 2004; 5:203-211.

9. Martin A, Nolan D, Mallal S. HLA-B ${ }^{\star} 5701$ typing by sequencespecific amplification: validation and comparison with sequence-based typing. Tissue Antigens 2005; 65:571-574.

10. Minitab ${ }^{\circledR}$ Statistical Software: http//www.minitab.com (last accessed 01/09/09).

11. New Allele Frequency Database: http://www.allelefrequencies. net. Middleton D., Menchaca L., Rood H., Komerofsky R. Tissue Antigens 2003; 61:403-407.

12. Encina F. Historia de Chile. Capitulo III. Santiago, Chile: Sociedad editora Revista Ercilla, 1983.

13. Cifuentes L, Morales R, Sepúlveda D et al. DYS19 and DYS199 loci in a Chilean population of mixed ancestry. Am J Phys Anthropol 2004; 125:85-89.

14. Migueles SA, Sabbaghian MS, Shupert WL et al. HLA B ${ }^{\star} 5701$ is highly associated with restriction of virus replication in a subgroup of HIV-infected long term nonprogressors. Proc Natl Acad Sci USA 2000; 97:2709-2714.

15. Horton H, Frank I, Baydo R et al. Preservation of $\mathrm{T}$ cell proliferation restricted by protective HLA alleles is critical for immune control of HIV-1 infection. J Immunol 2006; 177:7406-15 